\title{
Study on the Gain Material with Four Energy Level Model Using FDTD Method
}

\author{
Hui Xue ${ }^{1}$, Zhixiang Huang ${ }^{1}$, Xianliang $\mathrm{Wu}^{1,2}$ \\ ${ }^{1}$ Key Laboratory of Intelligent Computing and Signal Processing, Anhui University, Hefei, China \\ ${ }^{2}$ Department of Physics and Electronic Engineering Hefei Normal University, Hefei, China \\ Email: xuehui@ahu.edu.cn
}

Received 2013

\begin{abstract}
A faster numerical method based on FDTD for the four energy level atomic system is present here. The initial conditions for the electrons of each level are achieving while the fields are in steady state. Polarization equation, rate equations of electronic population and Maxwell's equations were used to describe the coupling between the atoms and electromagnetic wave. Numerical simulations, based on a finite-difference time-domain (FDTD) method, were utilized to obtain the population inversion and lasing threshold. The validity of the model and its theory is confirmed. The time, which we can observe the lasing phenomenon, is much shorter in our new model. Our model can be put into using in large scale simulations in mutiphysics to reduce the total simulated time.
\end{abstract}

Keywords: Finite-Difference Time-Domain; Gain Material; Lasing

\section{Introduction}

The system is always treated either semi classical or fully quantum mechanical [2, 3] while a high-frequency light is incident on a medium. Because of metallic nature of metamaterials constituent metamolecules, they suffer from high dissipative losses in the range of optical frequencies. Losses are too large in the real applications. It is better to incorporate the gain media into matematerial to compensate the losses. When an electromagnetic wave propagates in a medium, the dipole moment in the individual atom changes, in turn changing the total field coupling to the medium until a steady state established. A full-vectorial time domain approach is utilized to do self-consistent calculations. Finite-difference time-domain (FDTD) method [4] is used as a powerful tool in modeling linear dispersive media [5, 6]. In an attempt to achieve more realistic simulations two-level Maxwell-Bloch equations can be solved using iterative predictor-corrector finite difference time-domain FDTD methods to demonstrate saturation and self-induced transparency [7].

To simulate lasing dynamics, we present here a faster numerical simulation model for the four energy level atomic system. We use the populations of each level while the fields are achieving steady state as the initial value, in this way, the simulation time will sharply be reduced. The electromagnetic fields and atomic energy level populations at any time step can be calculated in terms of known quantities. Comparing the results of this model with those which putting all the electrons on the ground state level $\left(E_{0}\right)$. We can find that the results of two methods are similar. So the validity of the model and its theory is confirmed.

\section{Theoretical and Numerical Model}

\subsection{Rate Equations}

A simplified four level atomic system with energy levels $E_{0}, E_{1}, E_{2}, E_{3}$ and populations upon each level $N_{0}, N_{1}, N_{2}$, $\mathrm{N}_{3}$ is depicted in Figure 1.

In our model, the gain atoms are embedded in the each level of host medium aforehand. The electrons of the ground state level are pumped to the third level by the some external pumping mechanism $\left(P_{r}\right)$. After a very short time period $\tau_{32}$, the electrons of the third level $\left(E_{3}\right)$ fall into the second level $\left(E_{2}\right)$ by a non-radioactively transition. A population accumulates in the second level

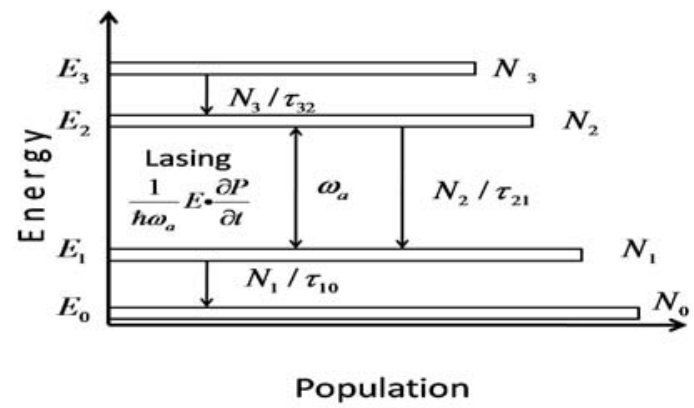

Figure 1. Schematic of the four-level atomic system model. 
due to the lifetime of the laser transition is longer than other lifetime, e.g. $\tau_{32}$. The amount of electrons in the second level $\left(E_{2}\right)$ is larger than the amount of electrons in the first level $\left(E_{1}\right)$.Then a population inversion is achieved. The lasing and optical amplification $E_{2}$ will happen at the frequency of $v_{21}=\left(E_{2}-E_{1}\right) / \hbar$. At last, the electrons transfer quickly and non-radiatively from the first state level $\left(E_{1}\right)$ to the ground state level $\left(E_{0}\right)$.

In the traditional four-level atomic system model, the populations can be modeled by the following rate equations:

$$
\begin{gathered}
\frac{\partial N_{3}}{\partial t}+\frac{N_{3}}{\tau_{32}}=P_{r} N_{0} \\
\frac{\partial N_{2}}{\partial t}-\frac{N_{3}}{\tau_{32}}+\frac{N_{2}}{\tau_{21}}=\frac{1}{\hbar \omega_{a}} E \cdot \frac{\partial P}{\partial t} \\
\frac{\partial N_{1}}{\partial t}-\frac{N_{2}}{\tau_{21}}+\frac{N_{1}}{\tau_{10}}=-\frac{1}{\hbar \omega_{a}} E \cdot \frac{\partial P}{\partial t} \\
\frac{\partial N_{1}}{\partial t}-\frac{N_{2}}{\tau_{21}}+\frac{N_{1}}{\tau_{10}}=-\frac{1}{\hbar \omega_{a}} E \cdot \frac{\partial P}{\partial t}
\end{gathered}
$$

We set the right of the equations as zero. The physical interpretation of this is owing to the steady state for the populations of each level. So the corresponding homogeneous equations can be rewritten as follows:

$$
\begin{aligned}
& \frac{\partial N_{3}}{\partial t}=-\frac{N_{3}}{\tau_{32}} \\
& \frac{\partial N_{2}}{\partial t}=\frac{N_{3}}{\tau_{32}}-\frac{N_{2}}{\tau_{21}} \\
& \frac{\partial N_{1}}{\partial t}=\frac{N_{2}}{\tau_{21}}-\frac{N_{1}}{\tau_{10}} \\
& \frac{\partial N_{0}}{\partial t}=\frac{N_{3}}{\tau_{32}}
\end{aligned}
$$

\subsection{Classical Electron Oscillator Model}

Using the Lorenz Model, the electric polarization in real atomic transitions can be described by the following equation:

$$
\frac{d^{2} P(t)}{d t^{2}}+\Delta \omega_{0} \frac{d P(t)}{d t}+\omega_{0}^{2} P(t)=-\frac{\gamma_{r}}{\gamma_{c}} \frac{e^{2}}{m} \Delta N(t) E(t)
$$

where $e$ is the charge of an electron. $m$ is the mass of an electron and $\Delta N=N_{2}-N_{1} \cdot \gamma_{\mathrm{r}}=1 / \tau_{21}$ is the real decay rate of the second level. $\gamma_{c}=1 / \tau_{21}$ is the classical rate. $\Delta \omega_{0}$ is the total energy decayrate and $\Delta \omega_{0}=1 / \tau_{21}+2 / T_{2} . T_{2}$ is the mean time between dephasing events.

\subsection{FDTD Formulation}

Presuming a two-dimensional problem of a plane wave propagating along $+y$ direction, using spatial and temporal interleaving of the fields and the central differencing scheme. We can write the discretized equations for the electric and magnetic fields as follows:

$$
\begin{aligned}
& H_{x}^{n+\frac{1}{2}}\left(i, j+\frac{1}{2}\right)-H_{x}^{n-\frac{1}{2}}\left(i, j+\frac{1}{2}\right)= \\
& -\frac{\Delta t}{\mu_{0} \Delta y}\left[E_{z}^{n}(i, j+1)-E_{z}^{n}(i, j)\right] \\
& H_{y}^{n+\frac{1}{2}}\left(i+\frac{1}{2}, j\right)-H_{y}^{n-\frac{1}{2}}\left(i+\frac{1}{2}, j\right) \\
& =\frac{\Delta t}{\mu_{0} \Delta y}\left[E_{z}^{n}(i+1, j)-E_{z}^{n}(i, j)\right] \\
& E_{z}^{n+1}(i+1, j)-E_{z}^{n}(i+1, j)= \\
& -\frac{P_{z}^{n+1}(i, j)-P_{z}^{n}(i, j)}{\varepsilon} \\
& +\frac{\Delta t}{\varepsilon \Delta x}\left[H_{y}^{n+\frac{1}{2}}\left(i+\frac{1}{2}, j\right)-H_{y}^{n+\frac{1}{2}}\left(i-\frac{1}{2}, j\right)\right] \\
& -\frac{\Delta t}{\varepsilon \Delta y}\left[H_{x}^{n+\frac{1}{2}}\left(i, j+\frac{1}{2}\right)-H_{x}^{n+\frac{1}{2}}\left(i, j-\frac{1}{2}\right)\right]
\end{aligned}
$$

The macroscopic polarization has a nonzero component in the $x$ direction alone. The polarization equation can be discretized as:

$$
\begin{aligned}
& P_{z}^{n+1}(i+1, j)=\frac{2-\omega_{0}^{2} \Delta t^{2}}{1+\Delta \omega_{0} \Delta t / 2} P_{z}^{n}(i, j) \\
& -\frac{1-\Delta \omega_{0} \Delta t / 2}{1+\Delta \omega_{0} \Delta t / 2} P_{z}^{n-1}(i, j) \\
& +\frac{\frac{\gamma_{r}}{\gamma_{c}} \frac{e^{2}}{m} \Delta t^{2}}{1+\Delta \omega_{0} \Delta t / 2}\left[N_{1}^{n}(i, j)-N_{2}^{n}(i, j)\right] E_{z}^{n}(i, j)
\end{aligned}
$$

Similarly, the discrete rate equations are:

$$
\begin{aligned}
& N_{3}^{n+1}(i, j)=\frac{2 \tau_{32}-\Delta t}{2 \tau_{32}+\Delta t} N_{3}^{n}(i, j) \\
& +P_{r} \frac{2 \tau_{32}}{2 \tau_{32}+\Delta t} N_{0}^{n}(i, j) \\
& N_{2}^{n+1}(i, j)=\frac{2 \tau_{21}-\Delta t}{2 \tau_{21}+\Delta t} N_{2}^{n}(i, j) \\
& +\frac{\tau_{21} \Delta t}{\tau_{32}\left(\tau_{21}-\Delta t\right)}\left[N_{3}^{n+1}(i, j)+N_{3}^{n}(i, j)\right] \\
& +\frac{\tau_{21}}{\hbar \omega_{0}\left(2 \tau_{21}+\Delta t\right)}\left[E_{z}^{n+1}(i, j)+E_{z}^{n}(i, j)\right]\left[P_{z}^{n+1}(i, j)-P_{z}^{n}(i, j)\right] \\
& N_{1}^{n+1}(i, j)=\frac{2 \tau_{10}-\Delta t}{2 \tau_{10}+\Delta t} N_{1}^{n}(i, j) \\
& +\frac{\tau_{10} \Delta t}{\tau_{21}\left(2 \tau_{10}+\Delta t\right)}\left[N_{2}^{n+1}(i, j)+N_{2}^{n}(i, j)\right] \\
& +\frac{\tau_{10}}{\hbar \omega_{0}\left(2 \tau_{10}+\Delta t\right)}\left[E_{z}^{n+1}(i, j)+E_{z}^{n}(i, j)\right]\left[P_{z}^{n+1}(i, j)-P_{z}^{n}(i, j)\right]
\end{aligned}
$$




$$
\begin{aligned}
& N_{0}^{n+1}(i, j)=\frac{\Delta t}{2 \tau_{10}}\left[N_{1}^{n+1}(i, j)+N_{1}^{n}(i, j)\right] \\
& +\left(1-\Delta t P_{r}\right) N_{0}^{n}(i, j)
\end{aligned}
$$

\section{Numerical Results}

The 2D system incorporated a gain material as showing in Figure 2.

By solving the differential equations (1a)-(1d) the amount of electrons of each level in each time step can be gotten as (8a)-(8b):

$$
\begin{gathered}
N_{3}(t)=c_{0} e^{-t / \tau_{32}} \\
N_{2}(t)=c_{1} e^{-t / \tau_{21}}-\frac{c_{0} \tau_{21}}{\tau_{21}-\tau_{32}} e^{-t / \tau_{32}} \\
N_{1}(t)=c_{2} e^{-t / \tau_{10}}-\frac{c_{1} \tau_{10}}{\tau_{10}-\tau_{21}} e^{-t / \tau_{21}} \\
+\frac{c_{0} \tau_{10} \tau_{32}}{\left(\tau_{21}-\tau_{32}\right)\left(\tau_{10}-\tau_{32}\right)} e^{-t / \tau_{32}} \\
N_{0}(t)=c_{3}-c_{2} e^{-t / \tau_{10}}+\frac{c_{1} \tau_{21}}{\tau_{10}-\tau_{21}} e^{-t / \tau_{21}} \\
-\frac{c_{0} \tau_{32}^{2}}{\left(\tau_{21}-\tau_{32}\right)\left(\tau_{10}-\tau_{32}\right)} e^{-t / \tau_{32}}
\end{gathered}
$$

where

$$
\begin{aligned}
& c_{0}=N \tau_{10} P_{r} \cdot\left(\left(\tau_{10}+\tau_{21}+\tau_{32}\right) P_{r}+1\right)^{-1} \\
& c_{1}=\frac{c_{0} \tau_{21}}{\tau_{21}-\tau_{10}}+N \tau_{21} P_{r} \cdot\left(\left(\tau_{10}+\tau_{21}+\tau_{32}\right) P_{r}+1\right)^{-1} \\
& c_{2}=\frac{c_{1} \tau_{10}}{\tau_{10}-\tau_{21}}+N \tau_{10} P_{r} \cdot\left(\left(\tau_{10}+\tau_{21}+\tau_{32}\right) P_{r}+1\right)^{-1} \\
& c_{3}=c_{2}-\frac{c_{1} \tau_{21}}{\tau_{10}-\tau_{21}}-\frac{c_{0} \tau_{10}}{\left(\tau_{21}-\tau_{10}\right)} \\
& +N \cdot\left(\left(\tau_{10}+\tau_{21}+\tau_{32}\right) P_{r}+1\right)^{-1}
\end{aligned}
$$
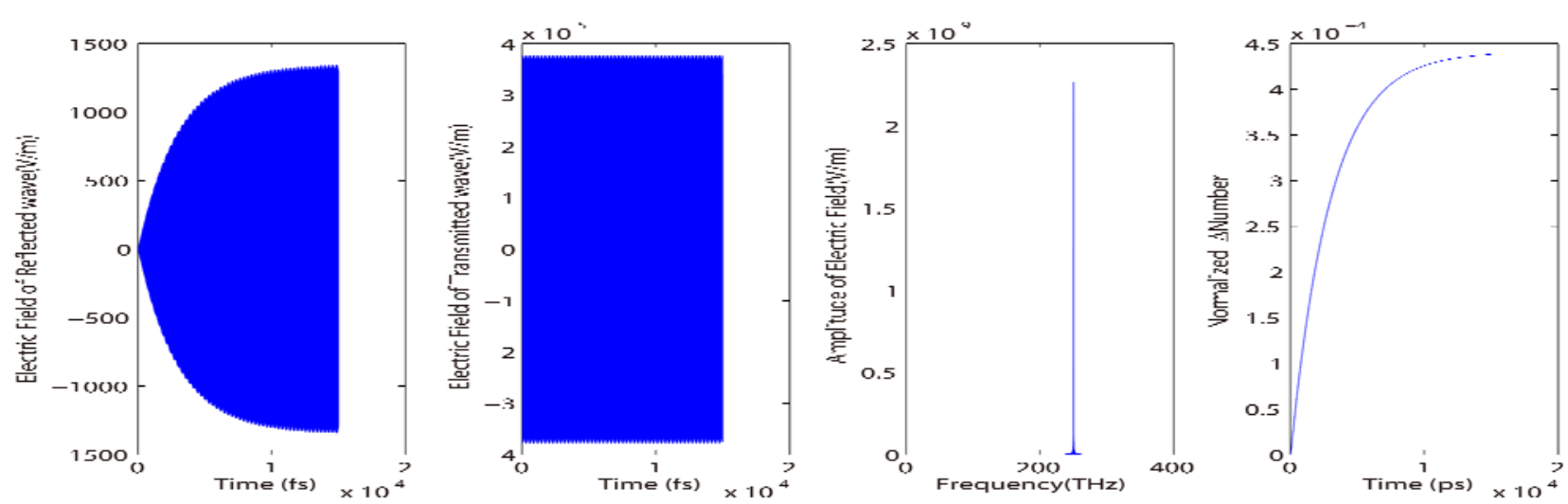

(a)The situation when all the electrons are embedded on the level $\left(E_{0}\right)$.The pump rate is $9 \times 10^{7} 1 / \mathrm{s}$.

The initial state is $t=0$. So we can embed the gain atoms in the each level of host medium aforehand base on above equations. There is no polarization at first. However, the system begins to evolve while electrons are pumped by external pumping into high energy level.

We set parameters as fellows. The slab width is 300 $\mathrm{nm}$. The gain slab width is $100 \mathrm{~nm}$. The discrete time and space steps are chosen as $\Delta t=1.667 \times 10^{-17} \mathrm{~s}$ and $\Delta x=1$ $\times 10^{-8} \mathrm{~m}$. The center frequency of the radiation $\omega_{0}$ is set as $2 \pi \times 10^{14} \mathrm{~Hz}$. $\Delta \omega_{0}$ is set as $5 \mathrm{THz}$. The parameters $\tau_{10}$, $\tau_{21}$ and $\tau_{32}$ are chosen as $5 \times 10^{-14} \mathrm{~s}, 5 \times 10^{-12} \mathrm{~s}$ and $5 \times$ $10^{-14} \mathrm{~s}$. The total electron density $\left(N_{t}\right)$ is chosen as $6 \times$ $10^{23} \mathrm{~m}^{-3}$.The total iterated time step is set as $1.5 \times 10^{6}$. The frequency of the CW wave is $250 \mathrm{THz}$.

We first set the pump rate $\left(P_{r}\right)$ as $9 \times 10^{7} 1 / \mathrm{s}$. A continuous wave is generated. We then let it propagate through the gain slab. We may find from the Figures 3 (a) and (b) that there is no lasing. As we increase the pump rate $\left(P_{r}\right)$, the lasing phenomenon appear, see Figures 3(c) and (d). With the pump rate increases (Figures 3(e) and (f)), the peak becomes larger. The Figures 3(a), (c) and (e) are the situations when all the electrons are embedded on the level $\left(E_{0}\right)$. The Figures 3(b), (d) and (f) are the situations when electrons are embedded on each level base on the equations (7a)-(7d).

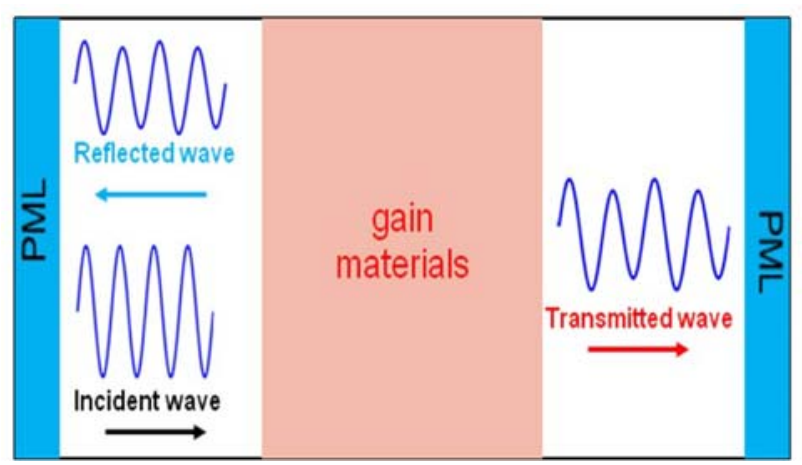

Figure 2. Schematic of the simulated structure.

$$
\text { (n) }
$$

Copyright (C) 2013 SciRes. 

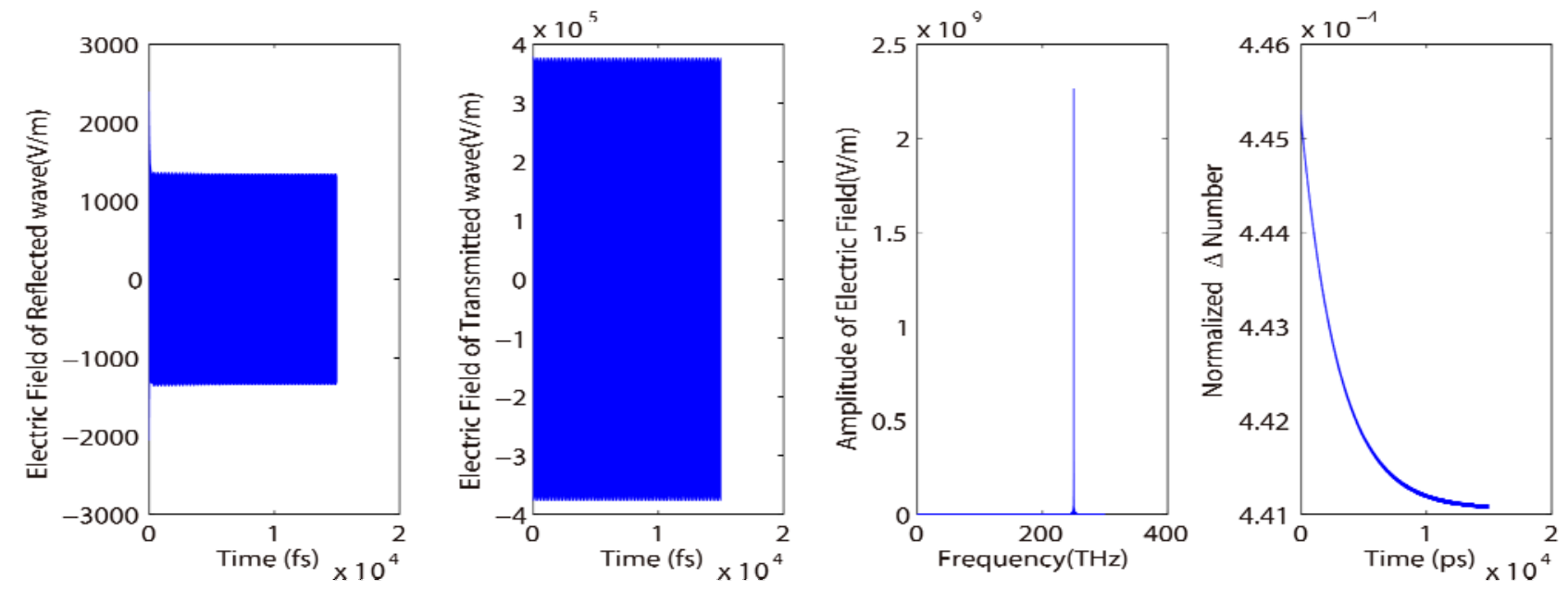

(b) The situation when electrons are embedded on each level base on the equations (7a) - (7d). The pump rate is $9 \times 10^{7} 1 / \mathrm{s}$.
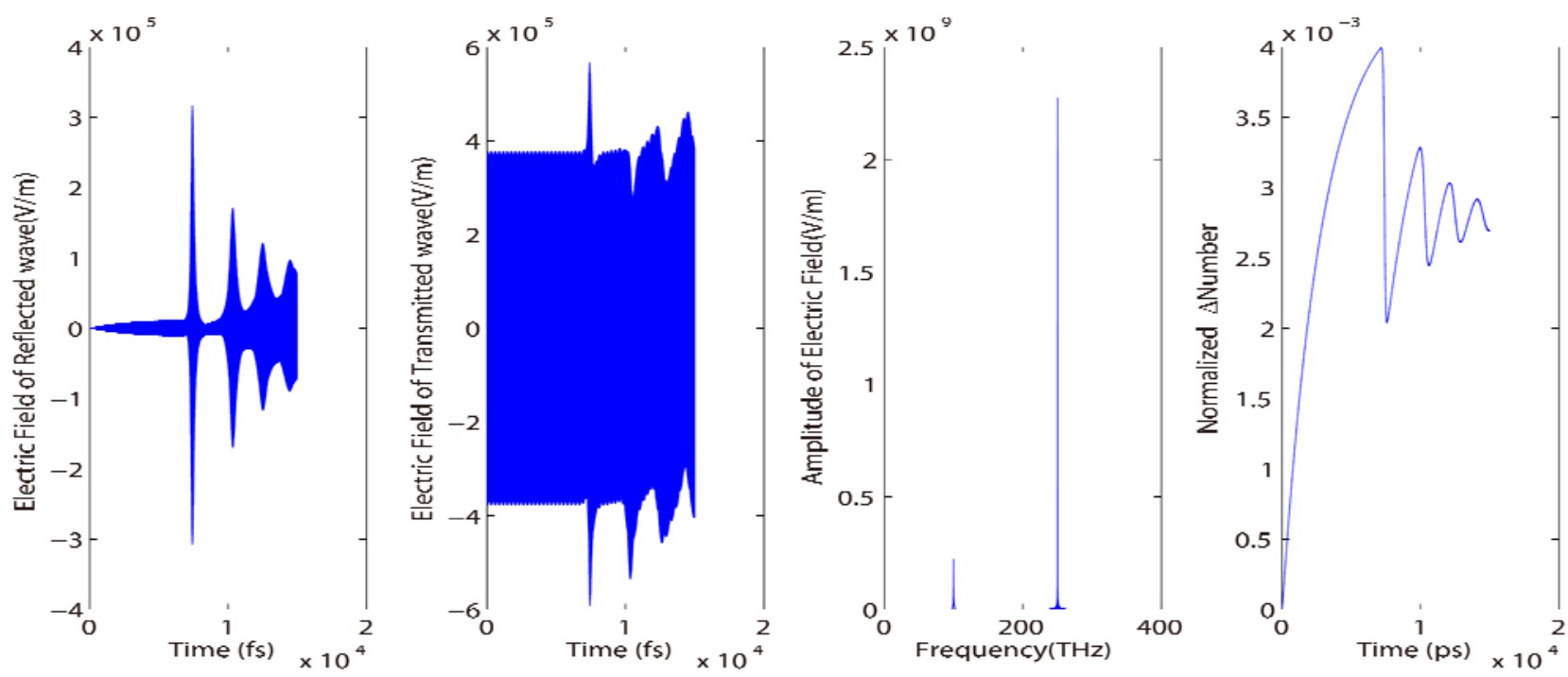

(c) The situation when all the electrons are embedded on the level $\left(E_{0}\right)$.The pump rate is $9 \times 10^{8} 1 / \mathrm{s}$.
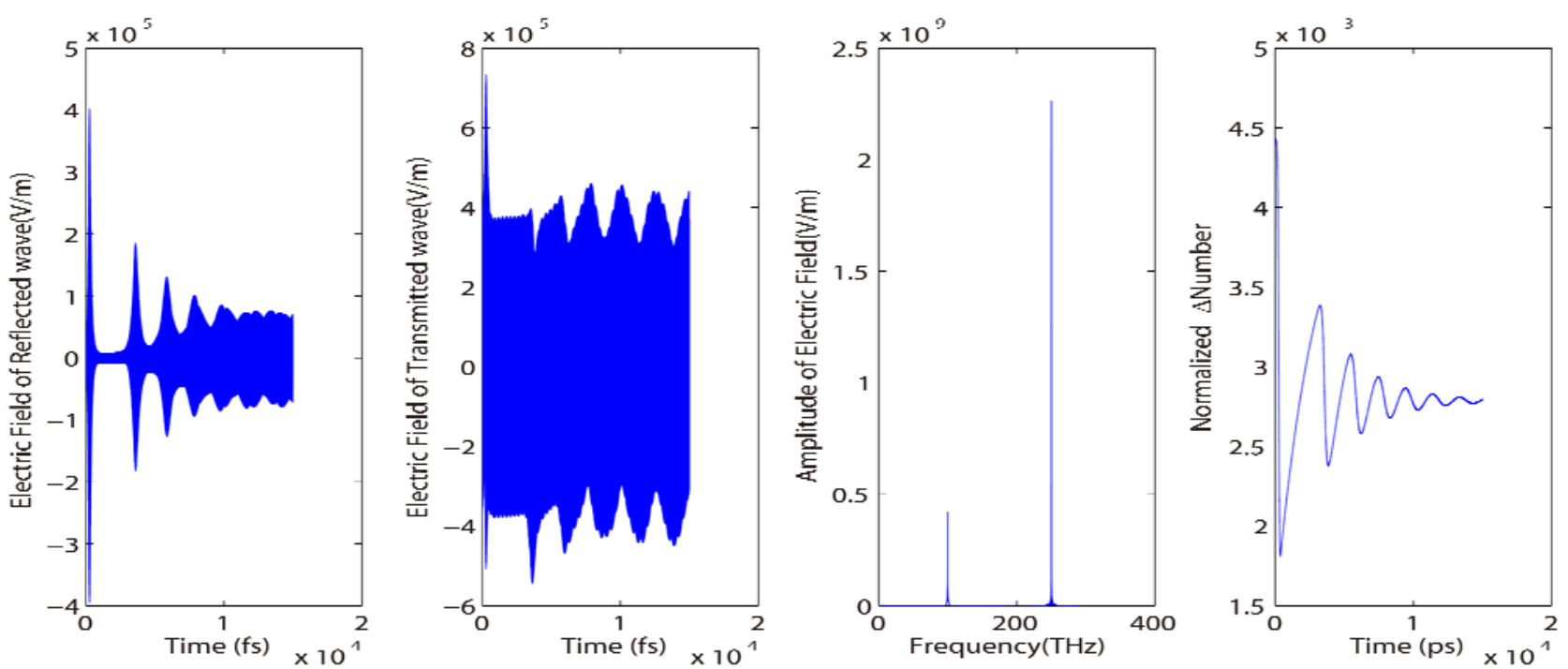

(d) The situation when electrons are embedded on each level base on the equations (7a) - (7d). The pump rate is $9 \times 10^{8} 1 / \mathrm{s}$. 

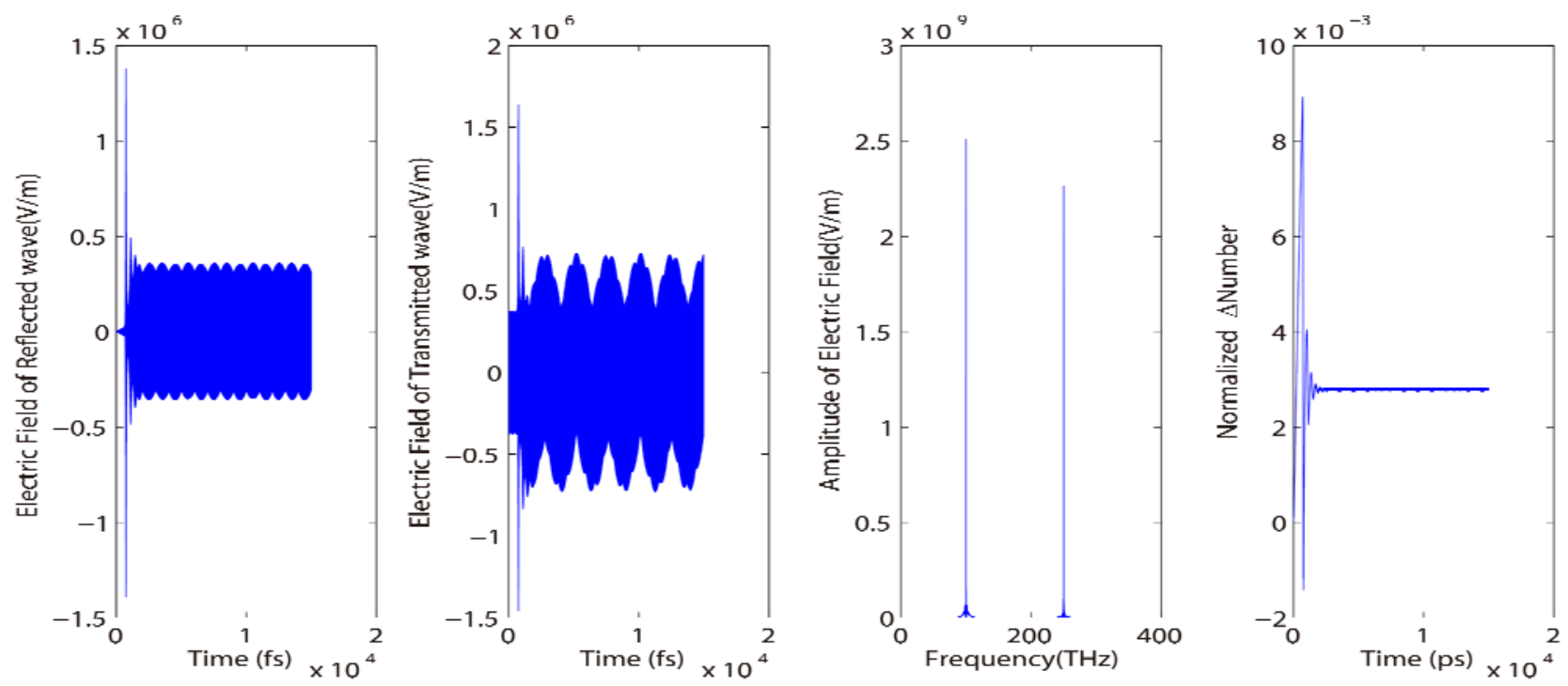

(e)The situation when all the electrons are embedded on the level $\left(E_{0}\right)$.The pump rate is $9 \times 10^{9} 1 / \mathrm{s}$.
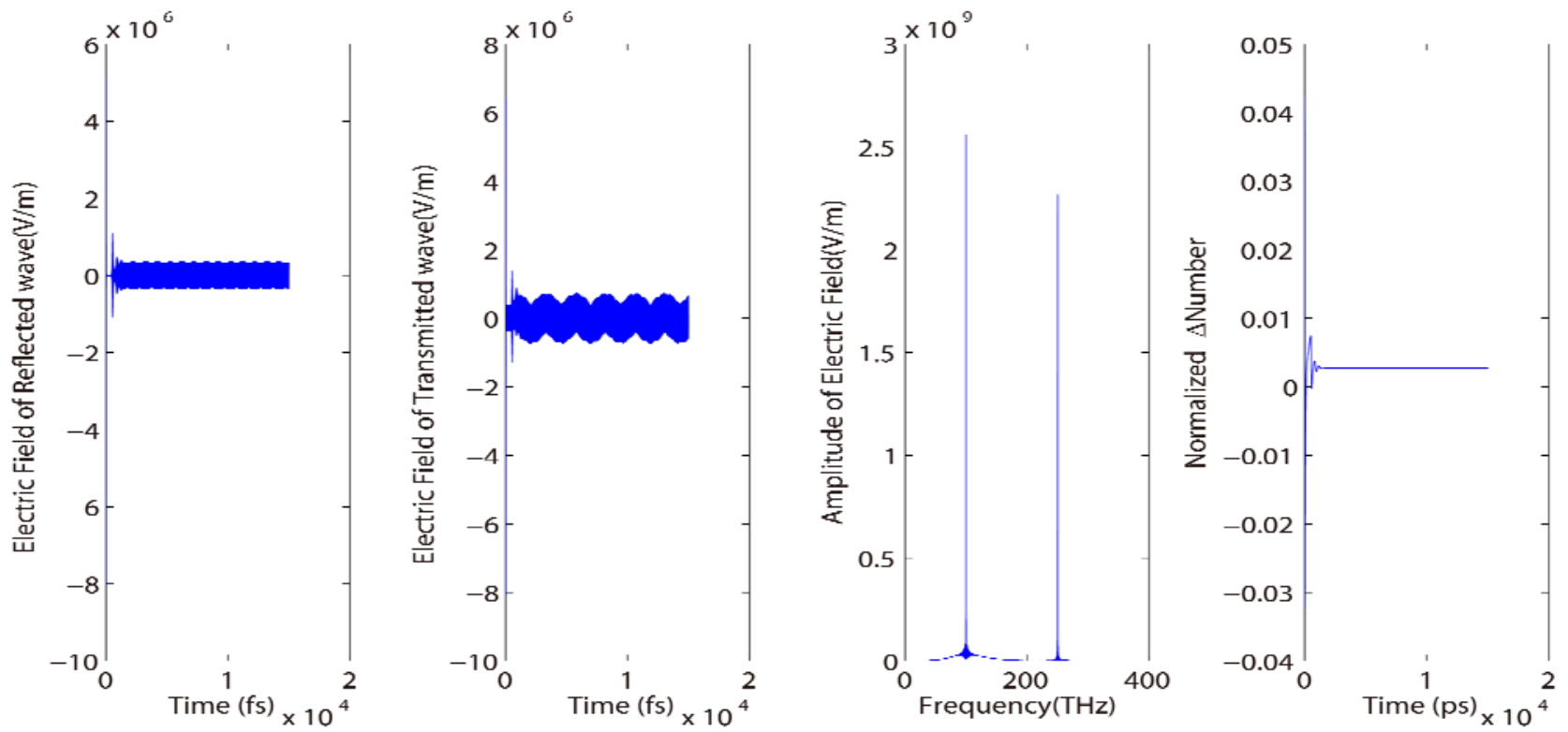

(f) The situation when electrons are embedded on each level base on the equations (7a) - (7d). The pump rate is $9 \times 10^{9} 1 / \mathrm{s}$.

Figure 3. The Electric field of the reflected wave, transmitted wave, the Fourier transform results, and the normalized difference of electron number between the upper level and lower level under different pump rate.

Populations changing can also be observed in the Figure 4. The Figure 4(a) is the situation when all the electrons are embedded on the ground state level, we may record this as case $a$. The Figure $\mathbf{4 ( b )}$ is the situation when electrons are embedded on each level base on the equations (7a)-(7d), namely case $b$. Here we use the pump rate of $9 \times 10^{8} 1 / \mathrm{s}$. If we increase the pump rate further, we may see the populations begin to oscillating strongly. It is easy to see that case $b$ has a shorter lasing time than the case $a$ does. That means our model costs less time to start to lase.
Figure 5 shows the comparisons of the lasing time under two situations, case $a$ and case $b$, with different pumping rates. We may find that the case $b$ needs less simulated time to lase than case $a$. Which means our new system takes less time to start to lase than the system which all the electrons are embedded on the ground level. Furthermore, when the pump rate gets higher the lasing time which the system begins to lase is decreasing.

\section{Conclusions}

A modified FDTD numerical model for gain materials 

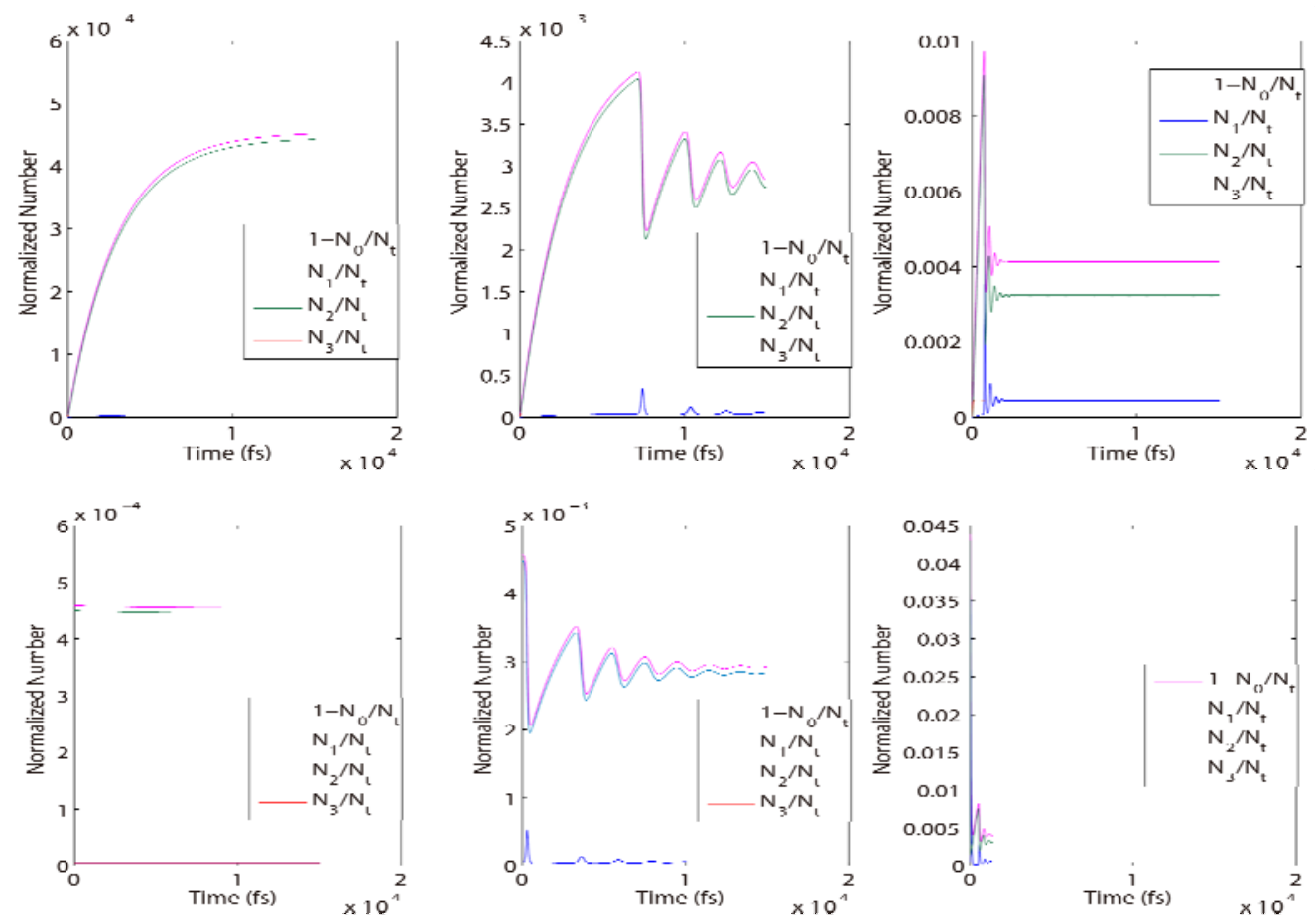

Figure 4. The two situations' populations change in each level with different pump rate.

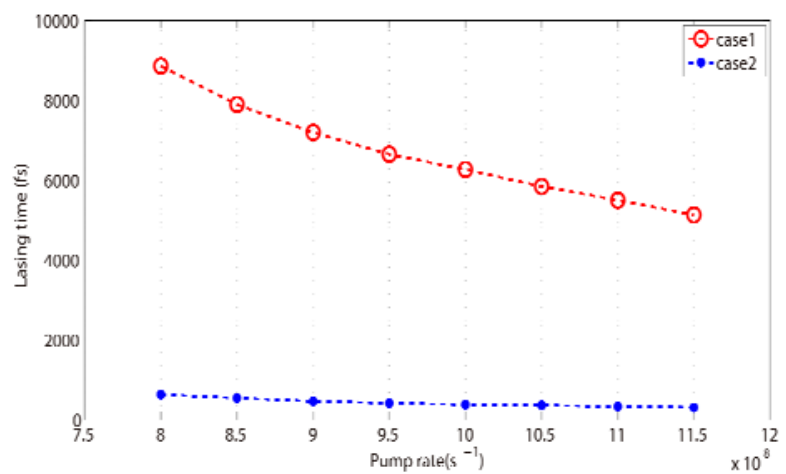

Figure 5. The lasing time of two situations under different pump rates.

and numerical simulations based on the lasing dynamics are presented here. The system begins to lase while the pumping rate increased to a certain value. Our modified model obviously reduces the lasing time (the time when the system start to lase) and the simulation results agree with the classic theory. Our model can be applied in larger electromagnetic and optics physics simulations to reduce the total simulated time.

\section{Acknowledgements}

This work was supported by the National Natural Science Foundation Of China under Grant (Nos.60931002, 61101064, 51277001), Distinguished Natural Science Foundation (No.1108085J01), Universities Natural Sci- ence Foundation of Anhui Province (Nos.KJ2011A002, KJ2012A013) and 211 Project of Anhui University.

\section{REFERENCES}

[1] Anan Fang, Thomas Koschny and Costas M Soukoulis, "Lasing in metamaterial nanostructures," Journal of Optics, Vol. 12, No. 2, 2010, p. 024013. doi:10.1088/2040-8978/12/2/024013

[2] J. Singh, "Semiconductor Optoelectronics: Physics and Technology,” McGraw-Hill, 1995.

[3] M.O. Scully and M.S. Zubairy, “Quantum Optics”, Cambridge University Press, Cambridge, England, 1997. doi:10.1017/CBO9780511813993

[4] A. Taflove, "Computational Electrodynamics: The FiniteDifference Time-Domain Method,” Artech House, 1995.

[5] P. Goorjian and A. Taflove, "Direct Time Integration of Maxwells Equations in Nonlinear Dispersive Media for Propagation and Scattering of Femtosecond Electromagnetic Solitons," Optics letters, Vol. 17 No. 3, 1992, pp. 180-182. doi:10.1364/OL.17.000180

[6] P. Goorjian, A. Taflove, R. Joseph and S. Hagness, "Computational Modeling of Femtosecond Optical Solitons from Maxwell's Equations," Quantum Electronics, Vol. 28, No. 10, 1992, pp. 2416-2422. doi:10.1109/3.159548

[7] R. Ziolkowski, J. Arnold and D. Gogny, "Ultrafast Pulse Interactions with Two-Level Atoms," Physical Review A, Vol. 52, No. 4, 1995, pp. 3082-3094. doi:10.1103/PhysRevA. 52.3082 